\section{THE CLEAREST CLINICAL OPTION}

Protective, award-winning eyewear that maintains complete safety whilst providing a lightweight, stylish solution for dental practitioners is possible with the Hogies range.

The range now offers improved comfort with an adjustable nosepiece which also helps to prevent fogging. A tough, hydrophobic coating protects the polycarbonate lens against solvents such as alcohol.

Clear vision is crucial for correct practice, and Hogies' ophthalmic- quality polycarbonate lens provides this while maintaining protection from high-velocity detritus and any contamination by chemicals or natural matter. Medical grade silicone in the Hogies Plus nose bridge, reinforced by stainless steel for strength and durability, allows improved adjustment and comfort for all facial requirements. Decreased fogging is achieved due to optimal airflow and with a choice of colours, Hogies Eyeguards remain the clearest option in clinical eye protection.

Reader response number 56

\section{PICK UP AND PRESS}

Swallow Dental Supplies offer a revolution in implant impression taking. The Miratray, an implant impression tray, uses patented foil technology - allowing you to create a high-precision impression in a matter of seconds.

Not only is the Miratray's patented foil technology easy to use, with the precision 'pick-up and press' technique, it also reduces the time necessary to take an impression. It also

\section{MAINTAIN ORIGINAL CAVITY ANATOMY}

DENTSPLY's NiTi alloy PathFile range of rotary instruments is ideal for quickly and safely creating a glide path and the preflaring of root canals without altering the original cavity anatomy.

Patients will experience a faster, more comfortable experience in the chair thanks to the reduced instrumentation time needed when using PathFile rotary instruments. PathFiles are flexible, resistant to cyclic fatigue and their gradual increase in diameter means that strong apical pressure is not required. PathFiles can be ordered immediately and come in three ISO sizes (013, 016 and 019). Reader response number 59

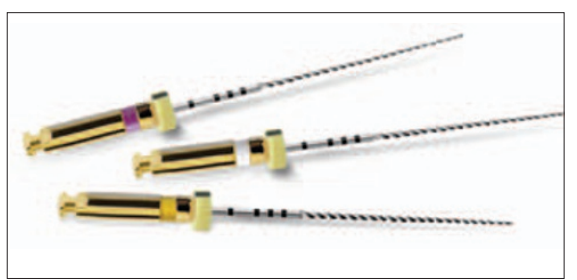

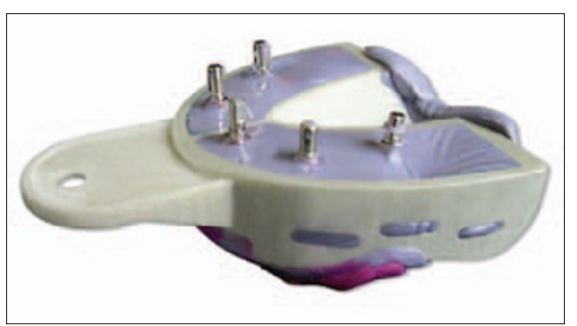

helps eliminate grinding of individual trays, reduces patient visits and saves money that ordinarily would go to a laboratory.

Reader response number 57

\section{IMAGING JUST FOR YOU}

The Dental Directory can help plan, deliver and install your new imaging system with the minimum disruption to your surgery.

Not only that, but The Dental Directory's independent position means that they are not tied to offer you any particular system. They offer the most comprehensive range of Digital Imaging systems available from the world's leading manufacturers such as Schick, Gendex, Satelec, E-Woo and Durr.

The range of choice ensures that they can find the system that's right for you.

Attractive finance packages are also available from leading institutions, so whatever your requirements are for a new or upgrade system - The Dental Directory can help. Reader response number 58 